\title{
The log log law for multidimensional stochastic integrals and diffusion processes
}

\section{Ludwig Arnold}

Let for $t \in[a, b] \subset[0, \infty)$

$$
x_{t}=c+\int_{a}^{t} f(s) d s+\int_{a}^{t} G(s) d w_{s},
$$

where $W_{s}$ is an n-dimensional Wiener process, $f(s)$ an $n$-vector process and $G(s)$ an $n \times m$ matrix process. $f$ and $G$ are nonanticipating and sample continuous. Then the set of limit points of the net

$$
\left\{\frac{x_{t+h}-x_{t}}{(2 h \log \log 1 / h)^{1 / 2}}\right\}_{e^{-1}>h+0}
$$

in $R^{n}$ is equal, almost surely, to the random ellipsoid $E_{t}=G(t) S_{m}, S_{m}=\left\{x \in R^{m}:|x| \leq 1\right\}$. The analogue of Lévy's law is also given. The results apply to $n$-dimensional diffusion processes which are solutions of stochastic differential equations, thus extending the versions of Hinčin's and Lévy's laws proved by H.P. Mckean, Jr, and W.J. Anderson.

\section{Introduction}

The local behavior of the paths of an m-dimensional standard Wiener

Received 16 June 1971. 
process $W_{t}$ is described by Hinčin's local log log law and Lévy's law concerning the modulus of continuity. The $\log \log$ law states that for fixed $t \geq 0$ the set of limit points of the net

$$
\left\{\frac{w_{t+h}{ }^{-W_{t}}}{(2 h \log \log 1 / h)^{1 / 2}}\right\}_{e^{-1}>h \downarrow 0}
$$

is equal, almost surely, to the m-dimensional closed unit sphere $S_{m}$, while Lévy's law states that the limit points of

$$
\left\{\frac{W_{t+h^{-}} W^{1 / 2}}{(2 h \log I / h)^{1 / h+0, a \leq t \leq b-h}},[a, b] \subset[0, \infty),\right.
$$

coincide, almost surely, with $S_{m}$. In the usual formulation of these laws, only the limit point of maximum modulus 1 is mentioned.

A random function on $[a, b] \subset[0, \infty)$ is called nonanticipating if its value at $t$ is measurable with respect to a $\sigma$-algebra $F_{t}$, where $F_{t}$ contains all the events generated by $\left\{w_{s}, s \leq t\right\}$, is independent of $\left\{W_{s+t}{ }^{-W_{t}}, s \geq 0\right\}$, and satisfies $F_{t_{1}} \subset F_{t_{2}}\left(t_{1}<t_{2}\right)$. Let $f(t)$ be an $n$-vector process and $G(t)$ an $n \times m$ matrix process, $f$ and $G$ being nonanticipating and sample continuous. If $c$ is an n-vector random variable measurable with respect to $F_{a}$, then the process

$$
X_{t}=c+\int_{a}^{t} f(s) d s+\int_{a}^{t} G(s) d W_{s}, t \in[a, b],
$$

(where the first integral is an ordinary Riemann integral and the second one is a stochastic (Itô) integral) is well-defined, nonanticipating, and sample continuous. We are looking for the analogues of Hinčin's and Lévy's laws for the paths of $X_{t}$.

2. The main result

THEOREM. Let $x_{t}$ be the n-vector process defined by (1). Then 
(i) for fixed $t \in[a, b]$, the set of limit points of the net

$$
\left\{\frac{x_{t+h}-X_{t}}{(2 h \log \log 1 / h)^{1 / 2}}\right\}_{e^{-1}>h+0}
$$

is equal, almost surely, to the random closed ellipsoid

$$
E_{t}=G(t) S_{m}=\left\{x \in R^{n}: x=G(t) y, y \in S_{m}\right\} ;
$$

(ii) the set of limit points of

$$
\left\{\frac{x_{t+h^{-X}}}{(2 h \log 1 / h)^{1 / 2}}\right\}_{1>h \downarrow 0, a \leq t \leq b-h}
$$

is equal, almost surely, to the random closed set $\underset{a \leq t \leq b}{\cup} E_{t}$.

Proof. (i) Since $c+\int_{a}^{t} f(s) d s$ is continuously differentiable, it is enough to prove the result for the stochastic integral

$$
y_{t}=\int_{a}^{t} G(s) d W_{s}
$$

Write for fixed $t \in[a, b)$

$$
y_{t+h}-y_{t}=G(t)\left(w_{t+h}-w_{t}\right)+\int_{t}^{t+h}(G(s)-G(t)) d w_{s} .
$$

The limit points of

$$
\left\{\frac{G(t)\left(W_{t+h}-W_{t}\right)}{(2 h \log \log 1 / h)^{1 / 2}}\right\}_{h+0}
$$

are clearly, almost surely, equal to $G(t) S_{m}$. The proof will be completed once we know that

$$
\lim _{h \rightarrow 0} \frac{\int_{t}^{t+h}\left(G_{i j}(s)-G_{i j}(t)\right) d w_{s}^{j}}{(2 h \log \log 1 / h)^{1 / 2}}=0 \text { almost surely, } 1 \leq i \leq n, 1 \leq j \leq m .
$$


There exists a new scalar wiener process $\vec{w}_{t}^{i j}$ so that

$$
\int_{t}^{t+h}\left(G_{i j}(s)-G_{i j}(t)\right) d w_{s}^{j}=\bar{W}_{\tau(h)}^{i j}
$$

with

$$
\tau(h)=\int_{t}^{t+h}\left(G_{i j}(s)-G_{i j}(t)\right)^{2} d s,
$$

(see McKean [3], p. 29). Since by the scalar log log law

$$
\varlimsup_{h \downarrow 0} \frac{\left|\vec{W}_{\tau(h)}^{i j}\right|}{(2 \tau(h) \log \log 1 / \tau(h))^{1 / 2}}=1 \text { almost surely, }
$$

and

$$
\lim _{h \downarrow 0}\left(\frac{2 \tau(h) \log \log 1 / \tau(h)}{2 h \log \log 1 / h}\right)^{1 / 2}=\sqrt{\tau^{\prime}(0)}=0 \text { almost surely, }
$$

(see Anderson [1], Lemma 3.1.1), the result follows.

(ii) We proceed as in (i). Lévy's law for $w_{t}$ holds in any small interval, thus giving us as possible limit points of

$$
\left\{\frac{G(t)\left(w_{t+h}-w_{t}\right)}{(2 h \log 1 / h)^{1 / 2}}\right\}_{h \downarrow 0, a \leq t \leq b-n}
$$

the set $\underset{a \leq t \leq b}{U} E_{t}$. The remainder in (2) is treated as in (i) using Lévy's law for scalar processes instead of (3). Q.E.D.

We remark that the ellipsoid $E_{t}$ can also be written as

$$
E_{t}=U(t) \sqrt{\Lambda(t)} S_{n},
$$

where $G G^{T}=U \Lambda U^{T}, U$ being the $n \times n$ eigenvector matrix and $\Lambda$ the $n \times n$ diagonal matrix of the eigenvalues $\lambda_{i}$ of $G G^{T}$. Thus, $E_{t}$ is the ellipsoid whose main axes have length $\sqrt{\lambda_{i}}$ and point into the direction of the eigenvectors of $G G^{T}$. 
The theorem contains numerous special cases, in particular the statements

$$
\overline{l i m}_{h+0} \frac{\left|X_{t+h}-X_{t}\right|}{(2 h \log \log 1 / h)^{1 / 2}}=\max _{i} \sqrt{\lambda_{i}(t)}
$$

and

$$
\prod_{\substack{h+0 \\ a \leq t \leq b-h}} \frac{\left|x_{t+h}-X_{t}\right|}{(2 h \log 1 / h)^{1 / 2}}=\max _{a \leq t \leq b} \max _{i} \sqrt{\lambda_{i}(t)}
$$

proved for a particular case by McKean ([3], p. 96), and a result of Anderson [1] for the components $x_{t}^{i}$,

$$
\varlimsup_{h \downarrow 0} \frac{X_{t+h}^{i}-X_{t}^{i}}{(2 h \log \log 1 / h)^{1 / 2}}=\sqrt{\left(G(t) G(t)^{T}\right)_{i i}} .
$$

\section{Application to multidimensional diffusions}

If the vector stochastic differential equation

$$
d x_{t}=f\left(t, x_{t}\right) d t+G\left(t, x_{t}\right) d w_{t}, x_{a}=c \text {, }
$$

has continuous coefficients satisfying the usual Lipschitz condition and growth restriction, the solution is unique and constitutes a vector diffusion process $X_{t}$ (see Gikhman-Skorokhod [2], p. 402). Our theorem applies to $X_{t}$, where the random ellipsoid $E_{t}=G\left(t, X_{t}\right) S_{m}$ now depends only on $t$ and the state $X_{t}$.

\section{References}

[1] William James Anderson, "Local behaviour of solutions of stochastic integral equations", (Ph.D. thesis, McGill University, Montreal, $1969)$.

[2] I.I. Gikhman, A.V. Skorokhod, Introduction to the theory of random processes (Sounders, Philadelphia, London, Toronto, 1969). 
[3] H.P. McKean, Jr, Stochastic integrals (Academic Press, New York, London, 1969 ).

Centre de recherches mathématiques, Université de Montréal, Montréal, Canada. 